\title{
Relationship Among Health-Related Quality of Life and Autosomal Ancestry, Clinical and Socioeconomic Factors In Type 1 Diabetes in an Admixed Brazilian Population.
}

\section{Rossana Sousa Azulay}

Hospital Universitario da Universidade Federal do Maranhao

Débora Lago ( $\sim$ deby19lago@gmail.com )

Universidade Federal do Maranhão https://orcid.org/0000-0002-9311-5047

Glaucia Abreu Silva Santos

Hospital Universitario da Universidade Federal do Maranhao

Maria da Gloria Tavares

Hospital Universitario da Universidade Federal do Maranhao

\section{Vandilson Rodrigues}

Federal University of Maranhao: Universidade Federal do Maranhao

\section{Marcelo Magalhães}

Hospital Universitario da Universidade Federal do Maranhao

\section{Roberta Ferreira Reis}

Hospital Universitario da Universidade Federal do Maranhao

\section{Nayara Nunes}

Hospital Universitario da Universidade Federal do Maranhao

\section{Ana Gregória Ferreira Pereira de Almeida}

Hospital Universitario da Universidade Federal do Maranhao

\section{Adriana Guimarães Sá}

Research group in clinical and molecular endocrinology and metabology

\section{Gilvan Nascimento}

Hospital Universitario da Universidade Federal do Maranhao

\section{Sabrina Damianse}

Hospital Universitario da Universidade Federal do Maranhao

\section{Viviane Rocha}

Hospital Universitario da Universidade Federal do Maranhao

\section{Dayse Aparecida Silva}

Universidade do Estado do Rio de Janeiro

\section{Marília Brito Gomes}

Universidade do Estado do Rio de Janeiro 
Manuel Faria

Federal University of Maranhao: Universidade Federal do Maranhao

\section{Research}

Keywords: Diabetes mellitus type 1, Quality of life, Autosomal ancestry, Endocrine Diseases.

Posted Date: November 9th, 2021

DOl: https://doi.org/10.21203/rs.3.rs-970705/v1

License: (c) (1) This work is licensed under a Creative Commons Attribution 4.0 International License. Read Full License 


\section{Abstract}

BACKGROUND: Type 1 diabetes is a chronic disease of an autoimmune character that affects the quality of life of patients in different degrees. Race and socioeconomic differences directly affect glycemic control. This study aimed to evaluate the Health-related quality of life (HRQoL) of Type 1 diabetes mellitus (T1D) patients an admixed Brazilian population, and to identify the variables influencing this condition.

METHODS: This research is a cross-sectional study conducted at the University Hospital-Federal University of Maranhão Endocrinology Service with 152 T1D patients between 2017 and 2018. HRQoL information was obtained from two self-completed questionnaires: Short Form- 6 dimensions and EuroQol-5 dimensions with visual analog scale. For inference of autosomal ancestry, a panel of 46 autosomal informational insertion/deletion ancestry markers (AIM-Indels) was used. Demographic information, socioeconomic data, presence of chronic complications, glycemic control level, and type of treatment were collected.

RESULTS: In the study, the patients who had good HRQoL were characterized as follows: male, under 18 years old, single, with average of 11 years of schooling, had health insurance, with less than 5 years of diagnosis, practiced physical activity, experienced no hypoglycemia in the last 30 days, reported no chronic complications (retinopathy and nephropathy), participant in several group educational activities, used analogous insulin, monitored blood glucose, showed maximum treatment adherence, and came from the secondary service. Autosomal ancestry and self-reported color/race did not show influence on HRQoL indexes.

CONCLUSION: Our study is the first to measure the HRQoL, autosomal ancestry and recognize the impact of T1D on patients' lives in the State of Maranhão, Northeast of Brazil. The results validate the need to provide T1D patients with continuous training on self-management and self-monitoring, seeking better results in metabolic control and consequently, in the prevention of acute and chronic complications to generate positive impacts on the quality of life of this population. In addition, reinforcing physical activity at each appointment should be part of the health team's routine. We understand that ethnicity in a highly mixed population like ours did not influence the quality of life of these patients.

\section{Introduction}

Health-related quality of life (HRQoL) is defined as the value given to life, considering the functional impairments, the social repercussions induced by the disease state, complications, and treatments. This parameter also includes the political and economic organization of the health care system and the state of health (physical, psychological, and social), damages, symptoms, or disabilities. HRQoL incorporates the aspects involved in the health-disease process and the impact of such condition on the daily life of individuals (1)(2). 
Chronic diseases are the factors that most affect an individual's HRQoL, given that they permanently interfere with a person's lifestyle and limit their productive capacity and world vision. In this context, type 1 diabetes mellitus (T1D) is considered one of the diseases that most affects an individual's HRQoL, because the related therapy requires a radical change in the person and their family's lifestyle (3).

T1D is a chronic disease and occurs due to autoimmune destruction of $\beta$ cells, leading to insulin deficiency and a lifelong need for insulin; it may cause serious short- and long-term complications. The incidence of T1D shows accentuated geographic variation, with rates varying from 38.4 in Finland, 7.6 in Brazil, and 0.5 in Korea for every 100 thousand individuals under 15 years of age. The incidence of T1D in the city of Bauru-SP between 1986 and 2006 was 13.7/100,000 (4). This incidence increased globally at a rate of approximately $3 \%$ per year (5).

T1D affects the psychological and emotional well-being of patients and their families(6). The metabolic control of diabetes and the improvement of HRQoL of the patients are equally important in preventing complications (7) From childhood onset to young adulthood, lifelong treatment, and special care are required to prevent or delay the short- and long-term complications of T1D(8). Current guidelines and studies indicate that diabetes care should be individualized for each patient, and a healthy lifestyle can improve glycemic control and decrease the risk of complications (9).

Race and socioeconomic differences are also implicated in T1D control. Although T1D is more frequent among white non-Hispanics when compared to Africans and Hispanics, African and Hispanic children and adolescents have worse control when compared to their white peers (10).

This study aimed to evaluate the HRQoL of T1D patients from State of Maranhão and correlated with autosomal ancestry, clinical and socioeconomic factors.

\section{Methodology}

\subsection{Study design}

This research is a cross-sectional study conducted at the Endocrinology Service of University HospitalFederal University of Maranhão (HU-UFMA), a reference service for the care of T1D patients in the State of Maranhão. The patients who enrolled at HU-UFMA, met the inclusion criteria, and accepted to participate in the study were admitted by reading and signing an informed consent form. The consultations were performed for 18 months starting from the date of study approval by the Research Ethics Committee of the University Hospital of the Federal University of Maranhão-CEP/HU-UFMA.

\subsection{Sample and eligibility criteria}

The study population consisted of T1D patients who used the specific outpatient clinic for T1D, called tertiary care service, at the UFMA High-complexity University Hospital, other non-specific endocrinology outpatient clinics for T1D, and considered secondary care services (specialized centers). With support 
from a diabetes specialist assigned in São Luís, State of Maranhão, the patients were referred for interview and spontaneous data collection during their routine consultations.

T1D patients older than 10 years old were included. We excluded patients who presented in the three months prior to the history of acute infectious disease or diabetic ketoacidosis, pregnancy and lactation.

\subsection{Data collection}

The T1D patients underwent a clinical demographic survey using a standardized questionnaire for data collection. The demographic data included gender, age (years), self-reported color/race, age at diagnosis (years), duration of T1D (years), level of physical activity, education, family income, availability of health insurance, participation in an educational group, insulin regimen used, and occurrence of hypoglycemia in the last 30 days.

The clinical variables evaluated were as follows: weight $(\mathrm{kg})$, height $(\mathrm{cm})$, body mass index (BMI), and blood pressure $(\mathrm{mmHg})$. Glycated hemoglobin (A1c) was determined by high performance liquid chromatography (reference value: $4.0-6.0 \%$ ), and albuminuria was screened by immunoturbidimetry of random urine sample collection on three occasions.

The HRQoL was assessed by the EuroQol questionnaire, which includes two tools: EuroQol-5 dimensions (EQ-5D) and EuroQol with visual analog scale (EQ-VAS). The first descriptively analyzes five problem dimensions (mobility, self-care, usual activities, pain and discomfort, anxiety, and depression) on a threescore scale graded 1 to 3 (1: "I have no problems"; 2 : "I have some problems"; 3 : "I have extreme problems"). The EQ-VAS (general health status) consists of an analog scale from 0 (very poor health status) to 100 (optimal health status) which was used by the patients to verify or report a value that reflects their perception of their health status (11)(12).

HRQoL was also evaluated by Short Form-6 dimensions (SF-6D), which was adapted for use in Brazil and used to describe the health status and generate utility indices derived from SF-36 items. This questionnaire comprises six domains with 4 to 6 levels and can generate 18,000 health states. The algorithm adapted for Brazil was used to calculate the utility scores for each health status identified after SF-6D application. The dimensions evaluated in this questionnaire were as follows: functional capacity, global limitation, social aspect, pain, mental health, and vitality. The unique SF-6D score ranges from 0 to 1 and represents the strength of an individual's preference for a particular health status, with a score of zero indicating the worst health and one implying the best (13)(14).

The fundus of the eye was examined by indirect ophthalmoscopy (EYE TECH) under the effect of topical mydriatic medication. The fundus was classified as present or absent retinopathy, using the most affected eye for diagnosis (8).

ND was assessed by the estimated glomerular filtration rate (Chronic Kidney Disease Epidemiology Collaboration equations) and urinary albumin concentration. Microalbuminuria was defined as positive 
by the presence of urinary albumin in concentrations above $30 \mathrm{mg} / \mathrm{L}$ and negative at below $30 \mathrm{mg} / \mathrm{L}$ in at least two samples (normoalbuminuria reference value: $<30 \mathrm{mg} / \mathrm{L}$; microalbuminuria: $>30 \mathrm{mg} / \mathrm{L}$ ) (8)(15).

DNA extraction was performed on a peripheral blood sample using the SP QIA symphony commercial kit according to the manufacturer's guidelines (Qiagen, USA).

We analyzed autosomal ancestry using a panel of 46 autosomal informational insertion/deletion ancestry markers (AIM-Indels), amplified in a single multiplex PCR following the protocol described by Pereira et al. (16). The detection of polymorphisms in the generated fragments was performed by capillary electrophoresis in the ABI 3500 automatic sequencer (Life Technologies). Genotyping was achieved using GeneMapper Analysis Software v.4.1 (Life Technologies). Structure v.2.3.3 software was used to estimate ancestry.

\subsection{Statistical analysis}

The data were analyzed using STATA version 16.0 (Stata Corp., College Station TX, USA) and GraphPad Prism version 8 (GraphPad Software Inc., San Diego, USA). Continuous variables were expressed as mean \pm standard deviation (SD), and categorical variables were summarized as frequencies and percentages. A violin plot and a triangular plot were created displaying the distribution of the autosomal proportions of African, European and Amerindian ancestry of sample.

The Shapiro-Wilk test was used to assess the normality of continuous data. One-way analysis of variance (ANOVA) followed by Bonferroni post hoc test, Kruskal-Wallis test followed by Dunnet post hoc test and independent sample t-test were applied to analyze the data. The significance level adopted was $5 \%$.

\section{Results}

The study sample consisted of 152 T1D patients (79 males) with a mean age of $25.1 \pm 10.5$ years. The most declared self-reported color/race and marital status were brown (65.1\%) and single (75.7\%), respectively. The highest percentage of self-reported household income per capita income was US $192-$ 576 ( 1 - 3 Brazilian minimum wages) (61.9\%). Most of the patients reported having no health insurance $(74.21 \%)$. In the total sample, $52 \%$ of the patients were managed in a secondary health care unit (Table 1). 
Table 1

Distribution of sociodemographic data of the Type 1 diabetes patients.

\begin{tabular}{|c|c|c|}
\hline Variable & $n=152$ & (\%) \\
\hline \multicolumn{3}{|l|}{ Sex } \\
\hline Male & 79 & $(52.0)$ \\
\hline \multicolumn{3}{|l|}{ Age group } \\
\hline Up to 18 years & 52 & $(34.2)$ \\
\hline 19 years or older & 100 & $(65.8)$ \\
\hline \multicolumn{3}{|l|}{ Self-reported color/race } \\
\hline White & 44 & $(29.0)$ \\
\hline Black & 9 & $(5.9)$ \\
\hline Brown & 99 & $(65.1)$ \\
\hline \multicolumn{3}{|l|}{ Marital status } \\
\hline Single & 115 & $(75.7)$ \\
\hline Married & 31 & $(20.4)$ \\
\hline Divorced/ separated/ widowed & 6 & $(3.9)$ \\
\hline \multicolumn{3}{|l|}{ Education level (years) } \\
\hline $0-8$ years & 33 & $(21.7)$ \\
\hline $9-11$ years & 66 & $(43.4)$ \\
\hline 12 years or more & 53 & (34.9) \\
\hline \multicolumn{3}{|c|}{ Household income per capita (BMW) } \\
\hline Less than 1 & 11 & $(7.2)$ \\
\hline 1 to 3 & 94 & $(61.9)$ \\
\hline More than 3 & 40 & $(30.9)$ \\
\hline \multicolumn{3}{|l|}{ Private health insurance } \\
\hline Yes & 37 & $(24.3)$ \\
\hline No & 115 & $(75.7)$ \\
\hline \multicolumn{3}{|l|}{ Level of health care delivery } \\
\hline Secondary health care unit & 79 & $(52.0)$ \\
\hline Tertiary health care unit & 73 & $(48.0)$ \\
\hline
\end{tabular}




Variable $n=152$

(\%)

$\mathrm{BMW}=$ Brazilian minimum wages per month (1 Brazilian minimum wage was $\mathrm{R} \$ 954$ in 2018).

Table 2 describes the clinical characteristics and information on insulin use. The most striking variables were lack of physical activity, with $54.6 \%$ of the T1D patients answered their lack of participation in physical activity of any kind, $81.6 \%$ showed $\mathrm{HbA} 1 \mathrm{c} \geq 7 \%$ (the mean was $9.05 \pm 2.27 \mathrm{sd}$ ), $14.3 \%$ had retinopathy, and $15,8 \%$ had albuminuria $>30 \mathrm{~g} / \mathrm{L}$. 
Table 2

Distribution of clinical and therapy data of the Type 1 diabetes patients.

\begin{tabular}{|c|c|c|c|}
\hline Variable & mean \pm sd & $\mathbf{n}$ & $(\%)$ \\
\hline \multicolumn{4}{|c|}{ Time from diabetes diagnosis } \\
\hline$<5$ years & & 36 & $(23.7)$ \\
\hline$\geq 5$ years & & 116 & $(76.3)$ \\
\hline \multicolumn{4}{|c|}{ Age at diabetes diagnosis } \\
\hline$<5$ years & & 18 & $(11.8)$ \\
\hline $5-9$ years & & 35 & $(23.0)$ \\
\hline $10-18$ years & & 63 & $(41.5)$ \\
\hline$\geq 19$ years & & 36 & $(23.7)$ \\
\hline \multicolumn{4}{|l|}{ BMI category } \\
\hline Underweight & & 24 & $(15.8)$ \\
\hline Normal & & 96 & $(63.2)$ \\
\hline Overweight/ obesity & & 32 & $(21.0)$ \\
\hline \multicolumn{4}{|c|}{ Blood pressure (mmHg) } \\
\hline Diastolic & $115.9 \pm 18.7$ & & \\
\hline Systolic & $71.1 \pm 10.1$ & & \\
\hline \multicolumn{4}{|c|}{ Physical activity (days/week) } \\
\hline 0 day/week & & 83 & $(54.6)$ \\
\hline 1-2 days/week & & 23 & $(15.1)$ \\
\hline 3-7 days/week & & 46 & $(30.3)$ \\
\hline \multicolumn{4}{|c|}{ Hypoglycemia in the last month } \\
\hline Yes & & 95 & $(62.5)$ \\
\hline No & & 57 & $(37.5)$ \\
\hline \multicolumn{4}{|c|}{ Glycated hemoglobin A1c category (HbA1c) } \\
\hline$<7 \%$ & & 28 & $(18.4)$ \\
\hline$\geq 7 \%$ & & 124 & $(81.6)$ \\
\hline \multicolumn{4}{|l|}{ Diabetic Retinopathy* } \\
\hline Absent & & 60 & (85.7) \\
\hline
\end{tabular}




\begin{tabular}{|c|c|c|c|}
\hline Variable & mean \pm sd & $\mathbf{n}$ & $(\%)$ \\
\hline Present & & 10 & $(14.3)$ \\
\hline \multicolumn{4}{|l|}{ Kidney damage } \\
\hline Absent & & 128 & $(84.2)$ \\
\hline Present (Albuminuria > 30 mg/L) & & 24 & $(15.8)$ \\
\hline \multicolumn{4}{|l|}{ Diabetes support group } \\
\hline Yes & & 28 & $(18.4)$ \\
\hline No & & 124 & $(81.6)$ \\
\hline \multicolumn{4}{|l|}{ Intermediate/long-acting insulin } \\
\hline $\mathrm{NPH}$ & & 92 & $(60.5)$ \\
\hline Glargine/ Detemir & & 60 & $(39.5)$ \\
\hline \multicolumn{4}{|l|}{ Fast-acting insulin } \\
\hline Regular & & 70 & $(46.0)$ \\
\hline Lispro/ Aspart/ Glulisine & & 63 & $(41.5)$ \\
\hline No use & & 19 & $(12.5)$ \\
\hline \multicolumn{4}{|l|}{ Self-monitoring of blood glucose } \\
\hline Yes & & 124 & $(81.6)$ \\
\hline No & & 28 & $(18.4)$ \\
\hline \multicolumn{4}{|c|}{ Adherence of insulin self-administration } \\
\hline High adherence & & 14 & $(9.2)$ \\
\hline Medium adherence & & 85 & $(55.9)$ \\
\hline Low adherence & & 53 & (34.9) \\
\hline
\end{tabular}

The autosomal ancestry markers showed that European ancestry was higher than African and Native American ancestries in TD1 patients from Maranhão State (Figure 1a). The ancestral contributions were $46 \%$ European, 28\% African, and 24\% Native American. The triangular plot has shown that the T1D sample presented an admixed ancestry closer to European (Figure 1b).

The mean scores attributed to HRQoL in the T1D patients from Maranhão State were $0.76 \pm 0.18$ (SF-6D), $0.90 \pm 0.10$ (EQ-5D), and $75.6 \pm 21.2$ (EQ-VAS). The association between sociodemographic variables and health-related quality of life is shown in Table 3. The SF-6D index value was statistically higher in males, 
up to 18 years old, private health insurance users, and secondary health care unit users. EQ-5D index value was statistically higher in males, and people up to 18 years. EQ-VAS score was higher in people up to 18 years old, and single people. 
Table 3

Association between sociodemographic variables and health-related quality of life in type 1 diabetes patients.

\begin{tabular}{|c|c|c|c|c|c|c|}
\hline \multirow[t]{2}{*}{ Variable } & SF-6D & \multirow{2}{*}{$\begin{array}{l}P \\
\text { value }\end{array}$} & EQ-5D & \multirow{2}{*}{$\begin{array}{l}P \\
\text { value }\end{array}$} & EQ-VAS & \multirow{2}{*}{$\begin{array}{l}P \\
\text { value }\end{array}$} \\
\hline & mean \pm sd & & $\begin{array}{l}\text { mean } \pm \\
\text { sd }\end{array}$ & & mean \pm sd & \\
\hline Sex & & 0.005 & & 0.009 & & 0.215 \\
\hline Male & $0.80 \pm 0.17$ & & $\begin{array}{l}0.92 \pm \\
0.10\end{array}$ & & $\begin{array}{l}77.7 \pm \\
20.8\end{array}$ & \\
\hline Female & $0.71 \pm 0.19$ & & $\begin{array}{l}0.88 \pm \\
0.11\end{array}$ & & $\begin{array}{l}73.4 \pm \\
21.5\end{array}$ & \\
\hline Age group & & 0.001 & & 0.006 & & 0.001 \\
\hline Up to 18 years & $0.83 \pm 0.16$ & & $\begin{array}{l}0.93 \pm \\
0.08\end{array}$ & & $\begin{array}{l}83.0 \pm \\
18.6\end{array}$ & \\
\hline 19 years or older & $0.72 \pm 0.19$ & & $\begin{array}{l}0.88 \pm \\
0.11\end{array}$ & & $\begin{array}{l}71.8 \pm \\
21.5\end{array}$ & \\
\hline Self-reported color/race & & 0.658 & & 0.089 & & 0.229 \\
\hline White & $0.76 \pm 0.16$ & & $\begin{array}{l}0.92 \pm \\
0.09\end{array}$ & & $\begin{array}{l}73.8 \pm \\
20.3\end{array}$ & \\
\hline Black & $0.70 \pm 0.21$ & & $\begin{array}{l}0.83 \pm \\
0.08\end{array}$ & & $\begin{array}{l}65.6 \pm \\
18.6\end{array}$ & \\
\hline Brown & $0.76 \pm 0.19$ & & $\begin{array}{l}0.90 \pm \\
0.11\end{array}$ & & $\begin{array}{l}77.3 \pm \\
21.7\end{array}$ & \\
\hline Marital status & & 0.001 & & 0.243 & & 0.005 \\
\hline Single & $\begin{array}{l}0.78 \pm \\
0.18^{\mathrm{a}}\end{array}$ & & $\begin{array}{l}0.91 \pm \\
0.09\end{array}$ & & $\begin{array}{l}78.6 \pm \\
19.3^{\mathrm{a}}\end{array}$ & \\
\hline Married & $\begin{array}{l}0.71 \pm \\
0.16^{\mathrm{ab}}\end{array}$ & & $\begin{array}{l}0.86 \pm \\
0.14\end{array}$ & & $\begin{array}{l}68.0 \pm \\
22.5^{\mathrm{b}}\end{array}$ & \\
\hline Divorced/ separated/ widowed & $\begin{array}{l}0.53 \pm \\
0.23^{b}\end{array}$ & & $\begin{array}{l}0.87 \pm \\
0.13\end{array}$ & & $\begin{array}{l}58.3 \pm \\
33.1^{\mathrm{b}}\end{array}$ & \\
\hline Education level (years) & & 0.355 & & 0.864 & & 0.312 \\
\hline $0-8$ years & $0.78 \pm 0.22$ & & $\begin{array}{l}0.91 \pm \\
0.10\end{array}$ & & $\begin{array}{l}83.0 \pm \\
21.7\end{array}$ & \\
\hline $9-11$ years & $0.74 \pm 0.19$ & & $\begin{array}{l}0.90 \pm \\
0.10\end{array}$ & & $\begin{array}{l}74.2 \pm \\
22.5\end{array}$ & \\
\hline 12 years or more & $0.77 \pm 0.15$ & & $\begin{array}{l}0.90 \pm \\
0.10\end{array}$ & & $\begin{array}{l}72.8 \pm \\
18.4\end{array}$ & \\
\hline
\end{tabular}




\begin{tabular}{|c|c|c|c|c|c|c|}
\hline \multirow[t]{2}{*}{ Variable } & \multirow{2}{*}{$\begin{array}{l}S F-6 D \\
\text { mean } \pm \text { sd }\end{array}$} & \multirow{2}{*}{$\begin{array}{l}P \\
\text { value }\end{array}$} & \multirow{2}{*}{$\begin{array}{l}\text { EQ-5D } \\
\text { mean } \pm \\
\text { sd }\end{array}$} & \multirow{2}{*}{$\begin{array}{l}P \\
\text { value }\end{array}$} & EQ-VAS & \multirow{2}{*}{$\begin{array}{l}P \\
\text { value }\end{array}$} \\
\hline & & & & & mean $\pm s d$ & \\
\hline \multicolumn{2}{|l|}{ Head of the family } & \multicolumn{2}{|l|}{0.945} & \multicolumn{2}{|l|}{0.921} & \multirow[t]{2}{*}{0.884} \\
\hline Yes & \multicolumn{2}{|l|}{$0.76 \pm 0.18$} & \multicolumn{2}{|l|}{$\begin{array}{l}0.90 \pm \\
0.12\end{array}$} & $\begin{array}{l}76.1 \pm \\
19.5\end{array}$ & \\
\hline No & \multicolumn{2}{|l|}{$0.76 \pm 0.19$} & \multicolumn{2}{|l|}{$\begin{array}{l}0.90 \pm \\
0.10\end{array}$} & \multicolumn{2}{|l|}{$\begin{array}{l}75.5 \pm \\
21.8\end{array}$} \\
\hline \multicolumn{2}{|l|}{$\begin{array}{l}\text { Household income per capita } \\
\text { (BMW) }\end{array}$} & \multicolumn{2}{|l|}{0.605} & \multicolumn{2}{|l|}{0.716} & \multirow[t]{2}{*}{0.338} \\
\hline Less than 1 & \multicolumn{2}{|l|}{$0.70 \pm 0.19$} & \multicolumn{2}{|l|}{$\begin{array}{l}0.89 \pm \\
0.11\end{array}$} & $\begin{array}{l}80.0 \pm \\
15.4\end{array}$ & \\
\hline 1 to 3 & \multicolumn{2}{|l|}{$0.76 \pm 0.20$} & \multicolumn{2}{|l|}{$\begin{array}{l}0.91 \pm \\
0.11\end{array}$} & \multicolumn{2}{|l|}{$\begin{array}{l}76.9 \pm \\
22.0\end{array}$} \\
\hline More than 3 & \multicolumn{2}{|l|}{$0.76 \pm 0.16$} & \multicolumn{2}{|l|}{$\begin{array}{l}0.89 \pm \\
0.09\end{array}$} & \multicolumn{2}{|l|}{$\begin{array}{l}72.0 \pm \\
20.6\end{array}$} \\
\hline \multicolumn{2}{|l|}{ Private health insurance } & \multicolumn{2}{|l|}{0.025} & \multicolumn{2}{|l|}{0.208} & \multirow[t]{2}{*}{0.028} \\
\hline Yes & $0.81 \pm 0.19$ & & $\begin{array}{l}0.92 \pm \\
0.09\end{array}$ & & $\begin{array}{l}82.7 \pm \\
14.9\end{array}$ & \\
\hline No & $0.74 \pm 0.18$ & & $\begin{array}{l}0.89 \pm \\
0.11\end{array}$ & & $\begin{array}{l}73.4 \pm \\
22.4\end{array}$ & \\
\hline Level of health care delivery & & 0.032 & & 0.990 & & 0.048 \\
\hline Secondary health care unit & $0.79 \pm 0.18$ & & $\begin{array}{l}0.90 \pm \\
0.11\end{array}$ & & $\begin{array}{l}78.9 \pm \\
21.2\end{array}$ & \\
\hline Tertiary health care unit & $0.72 \pm 0.18$ & & $\begin{array}{l}0.90 \pm \\
0.09\end{array}$ & & $\begin{array}{l}72.1 \pm \\
20.8\end{array}$ & \\
\hline $\begin{array}{l}\text { SF-6D = Short-Form- } 6 \text { dimen } \\
\text { Europen quality of life-visual } \\
\text { wages per month ( } 1 \text { Brazilian } \\
\text { (one-way ANOVA, Kruskal-Wall } \\
\text { indicate significant differences } \\
\text { comparison test ( } P \text { values }<0 \text {. }\end{array}$ & $\begin{array}{l}\text { s. } E Q-5 D=E \\
\text { og scale. } \pm s \\
\text { num wage } \\
\text { dependent s } \\
\text { Neen catego }\end{array}$ & $\begin{array}{l}\text { uropean } \\
=\text { stand } \\
\text { a RS } 95 \\
\text { mple t-t } \mathrm{e} \\
\text { es using }\end{array}$ & $\begin{array}{l}\text { uality of li } \\
\text { d deviatic } \\
\text { in 2018). } \\
\text { t or Mann } \\
\text { 3onferroni }\end{array}$ & $\begin{array}{l}-5 \text { dime } \\
\text { BMW = } \\
\text { Id indica } \\
\text { hitney te } \\
\text { or Dunne }\end{array}$ & $\begin{array}{l}\text { ions. EQ-VA } \\
\text { azilian minin } \\
\text { es P values < } \\
\text { t). Different I } \\
\text { 's multiple }\end{array}$ & $\begin{array}{l}= \\
\text { um } \\
.05 \\
\text { tters }\end{array}$ \\
\hline
\end{tabular}

Table 4 shows the association between clinical variables and health-related quality of life. The SF-6D index was statistically higher in patients with from diabetes diagnosis $\geq 5$ years. Physical activity 3 or more days a week and no hypoglycemic episodes in the past month were associated with higher SF-6D and EQ-5D scores. Patients who present retinopathy had lower SF-6D and EQ-VAS indexes. The SF-6D index was lower in patients who had albuminuria. Patients who participated in the diabetes education 
group and using Glargine/ Detemir use and Lispro/ Aspart/ Glulisine presented higher HRQoL indexes. T1D patients who performed self-monitoring of blood glucose had higher EQ-5D and EQ-VAS indexes. 
Table 4

Association between clinical variables and health-related quality of life in type 1 diabetes patients.

\begin{tabular}{|c|c|c|c|c|c|c|}
\hline \multirow[t]{2}{*}{ Variable } & SF-6D & \multirow{2}{*}{$\begin{array}{l}P \\
\text { value }\end{array}$} & EQ-5D & \multirow{2}{*}{$\begin{array}{l}P \\
\text { value }\end{array}$} & EQ-VAS & \multirow{2}{*}{$\begin{array}{l}P \\
\text { value }\end{array}$} \\
\hline & mean $\pm s d$ & & mean $\pm s d$ & & mean \pm sd & \\
\hline Time from diabetes diagnosis & & 0.048 & & 0.588 & & 0.315 \\
\hline$\geq 5$ years & $\begin{array}{l}0.81 \pm \\
0.17\end{array}$ & & $\begin{array}{l}0.92 \pm \\
0.08\end{array}$ & & $\begin{array}{l}78.7 \pm \\
23.3\end{array}$ & \\
\hline$<5$ years & $\begin{array}{l}0.74 \pm \\
0.19\end{array}$ & & $\begin{array}{l}0.90 \pm \\
0.11\end{array}$ & & $\begin{array}{l}74.6 \pm \\
20.5\end{array}$ & \\
\hline Age at diabetes diagnosis & & 0.975 & & 0.233 & & 0.236 \\
\hline$<5$ years & $\begin{array}{l}0.75 \pm \\
0.16\end{array}$ & & $\begin{array}{l}0.89 \pm \\
0.10\end{array}$ & & $\begin{array}{l}78.3 \pm \\
18.3\end{array}$ & \\
\hline $5-9$ years & $\begin{array}{l}0.77 \pm \\
0.19\end{array}$ & & $\begin{array}{l}0.93 \pm \\
0.09\end{array}$ & & $\begin{array}{l}81.4 \pm \\
18.6\end{array}$ & \\
\hline $10-18$ years & $\begin{array}{l}0.76 \pm \\
0.20\end{array}$ & & $\begin{array}{l}0.88 \pm \\
0.12\end{array}$ & & $\begin{array}{l}73.4 \pm \\
22.4\end{array}$ & \\
\hline$\geq 19$ years & $\begin{array}{l}0.76 \pm \\
0.17\end{array}$ & & $\begin{array}{l}0.91 \pm \\
0.07\end{array}$ & & $\begin{array}{l}72.6 \pm \\
22.2\end{array}$ & \\
\hline BMI category & & 0.736 & & 0.177 & & 0.088 \\
\hline Underweight & $\begin{array}{l}0.78 \pm \\
0.21\end{array}$ & & $\begin{array}{l}0.93 \pm \\
0.09\end{array}$ & & $\begin{array}{l}82.9 \pm \\
19.2\end{array}$ & \\
\hline Normal & $\begin{array}{l}0.76 \pm \\
0.18\end{array}$ & & $\begin{array}{l}0.90 \pm \\
0.11\end{array}$ & & $\begin{array}{l}75.6 \pm \\
20.6\end{array}$ & \\
\hline Overweight/ obesity & $\begin{array}{l}0.74 \pm \\
0.18\end{array}$ & & $\begin{array}{l}0.89 \pm \\
0.07\end{array}$ & & $\begin{array}{l}70.3 \pm \\
23.2\end{array}$ & \\
\hline Physical activity (days/week) & & 0.023 & & 0.013 & & 0.216 \\
\hline 0 day/week & $\begin{array}{l}0.72 \pm \\
0.19^{a}\end{array}$ & & $\begin{array}{l}0.88 \pm \\
0.12^{\mathrm{a}}\end{array}$ & & $\begin{array}{l}73.3 \pm \\
22.1\end{array}$ & \\
\hline 1-2 days/week & $\begin{array}{l}0.81 \pm \\
0.18^{\mathrm{ab}}\end{array}$ & & $\begin{array}{l}0.94 \pm \\
0.09^{\mathrm{ab}}\end{array}$ & & $\begin{array}{l}81.9 \pm \\
17.1\end{array}$ & \\
\hline 3-7 days/week & $\begin{array}{l}0.80 \pm \\
0.16^{\mathrm{b}}\end{array}$ & & $\begin{array}{l}0.92 \pm \\
0.07^{b}\end{array}$ & & $\begin{array}{l}76.6 \pm \\
21.1\end{array}$ & \\
\hline Hypoglycemia in the last month & & 0.002 & & 0.024 & & 0.464 \\
\hline Yes & $\begin{array}{l}0.72 \pm \\
0.19\end{array}$ & & $\begin{array}{l}0.89 \pm \\
0.10\end{array}$ & & $\begin{array}{l}74.6 \pm \\
20.8\end{array}$ & \\
\hline No & $\begin{array}{l}0.82 \pm \\
0.15\end{array}$ & & $\begin{array}{l}0.92 \\
\pm 0.11\end{array}$ & & $\begin{array}{l}77.2 \pm \\
21.9\end{array}$ & \\
\hline
\end{tabular}




\begin{tabular}{|c|c|c|c|c|c|c|}
\hline \multirow[t]{2}{*}{ Variable } & \multirow{2}{*}{$\begin{array}{l}S F-6 D \\
\text { mean } \pm s d\end{array}$} & \multirow{2}{*}{$\begin{array}{l}P \\
\text { value }\end{array}$} & \multirow{2}{*}{$\begin{array}{l}E Q-5 D \\
\text { mean } \pm s d\end{array}$} & \multirow{2}{*}{$\begin{array}{l}P \\
\text { value }\end{array}$} & \multirow{2}{*}{$\begin{array}{l}\text { EQ-VAS } \\
\text { mean } \pm s d\end{array}$} & \multirow{2}{*}{$\begin{array}{l}\mathrm{P} \\
\text { value }\end{array}$} \\
\hline & & & & & & \\
\hline $\begin{array}{l}\text { Glycated hemoglobin A1c } \\
\text { category }\end{array}$ & & 0.514 & & 0.266 & & 0.684 \\
\hline$<7 \%$ & $\begin{array}{l}0.78 \pm \\
0.19\end{array}$ & & $\begin{array}{l}0.92 \pm \\
0.08\end{array}$ & & $\begin{array}{l}77.1 \pm \\
24.4\end{array}$ & \\
\hline$\geq 7 \%$ & $\begin{array}{l}0.75 \pm \\
0.18\end{array}$ & & $\begin{array}{l}0.90 \pm \\
0.11\end{array}$ & & $\begin{array}{l}75.3 \pm \\
20.5\end{array}$ & \\
\hline Diabetic Retinopathy & & 0.024 & & 0.127 & & 0.018 \\
\hline Absent & $\begin{array}{l}0.73 \pm \\
0.18\end{array}$ & & $\begin{array}{l}0.90 \pm \\
0.11\end{array}$ & & $\begin{array}{l}78.3 \pm \\
17.7\end{array}$ & \\
\hline Present & $\begin{array}{l}0.61 \pm \\
0.14\end{array}$ & & $\begin{array}{l}0.83 \pm \\
0.17\end{array}$ & & $\begin{array}{l}61.5 \pm \\
32.4\end{array}$ & \\
\hline Kidney damage & & 0.018 & & 0.144 & & 0.105 \\
\hline Absent & $\begin{array}{l}0.78 \pm \\
0.18\end{array}$ & & $\begin{array}{l}0.91 \pm \\
0.10\end{array}$ & & $\begin{array}{l}77.3 \pm \\
19.8\end{array}$ & \\
\hline $\begin{array}{l}\text { Present (Albuminuria }>30 \\
\mathrm{mg} / \mathrm{L} \text { ) }\end{array}$ & $\begin{array}{l}0.70 \pm \\
0.20\end{array}$ & & $\begin{array}{l}0.88 \pm \\
0.12\end{array}$ & & $\begin{array}{l}71.0 \pm \\
24.5\end{array}$ & \\
\hline Diabetes support group & & 0.021 & & 0.027 & & 0.001 \\
\hline Yes & $\begin{array}{l}0.83 \pm \\
0.17\end{array}$ & & $\begin{array}{l}0.94 \pm \\
0.07\end{array}$ & & $\begin{array}{l}86.9 \pm \\
13.9\end{array}$ & \\
\hline No & $\begin{array}{l}0.74 \pm \\
0.18\end{array}$ & & $\begin{array}{l}0.89 \pm \\
0.11\end{array}$ & & $\begin{array}{l}73.1 \pm \\
21.8\end{array}$ & \\
\hline $\begin{array}{l}\text { Intermediate/long-acting } \\
\text { insulin }\end{array}$ & & 0.002 & & 0.045 & & 0.001 \\
\hline $\mathrm{NPH}$ & $\begin{array}{l}0.72 \pm \\
0.19\end{array}$ & & $\begin{array}{l}0.89 \pm \\
0.11\end{array}$ & & $\begin{array}{l}71.0 \pm \\
22.9\end{array}$ & \\
\hline Glargine/ Detemir & $\begin{array}{l}0.82 \pm \\
0.17\end{array}$ & & $\begin{array}{l}0.92 \pm \\
0.08\end{array}$ & & $\begin{array}{l}82.6 \pm \\
16.0\end{array}$ & \\
\hline Fast-acting insulin & & $<0.001$ & & 0.009 & & 0.028 \\
\hline Regular & $\begin{array}{l}0.69 \pm \\
0.18^{\mathrm{a}}\end{array}$ & & $\begin{array}{l}0.88 \pm \\
0.11^{\mathrm{a}}\end{array}$ & & $\begin{array}{l}71.2 \pm \\
23.2^{\mathrm{a}}\end{array}$ & \\
\hline Lispro/ Aspart/ Glulisine & $\begin{array}{l}0.82 \pm \\
0.15^{\mathrm{b}}\end{array}$ & & $\begin{array}{l}0.93 \pm \\
0.07^{b}\end{array}$ & & $\begin{array}{l}80.5 \pm \\
16.7^{b}\end{array}$ & \\
\hline No use & $\begin{array}{l}0.82 \pm \\
0.20^{\mathrm{b}}\end{array}$ & & $\begin{array}{l}0.90 \pm \\
0.14^{\mathrm{ab}}\end{array}$ & & $\begin{array}{l}74.4 \pm \\
23.8^{\mathrm{ab}}\end{array}$ & \\
\hline
\end{tabular}




\begin{tabular}{|c|c|c|c|c|c|c|}
\hline \multirow[t]{2}{*}{ Variable } & \multirow{2}{*}{$\begin{array}{l}\text { SF-6D } \\
\text { mean } \pm s d\end{array}$} & \multirow{2}{*}{$\begin{array}{l}P \\
\text { value }\end{array}$} & EQ-5D & \multirow{2}{*}{$\begin{array}{l}P \\
\text { value }\end{array}$} & EQ-VAS & \multirow{2}{*}{$\begin{array}{l}P \\
\text { value }\end{array}$} \\
\hline & & & mean $\pm s d$ & & mean $\pm s d$ & \\
\hline $\begin{array}{l}\text { Self-monitoring of blood } \\
\text { glucose }\end{array}$ & & 0.091 & & 0.003 & & $<0.001$ \\
\hline Yes & $\begin{array}{l}0.77 \pm \\
0.18\end{array}$ & & $\begin{array}{l}0.92 \pm \\
0.08\end{array}$ & & $\begin{array}{l}79.2 \pm \\
17.5\end{array}$ & \\
\hline No & $\begin{array}{l}0.70 \pm \\
0.20\end{array}$ & & $\begin{array}{l}0.84 \pm \\
0.15\end{array}$ & & $\begin{array}{l}59.6 \pm \\
28.2\end{array}$ & \\
\hline $\begin{array}{l}\text { Adherence of insulin self- } \\
\text { administration }\end{array}$ & & 0.462 & & 0.688 & & 0.060 \\
\hline High adherence & $\begin{array}{l}0.77 \pm \\
0.23\end{array}$ & & $\begin{array}{l}0.92 \pm \\
0.11\end{array}$ & & $\begin{array}{l}77.8 \pm \\
30.1\end{array}$ & \\
\hline Medium adherence & $\begin{array}{l}0.77 \pm \\
0.18\end{array}$ & & $\begin{array}{l}0.90 \pm \\
0.10\end{array}$ & & $\begin{array}{l}78.0 \pm \\
19.5\end{array}$ & \\
\hline Low adherence & $\begin{array}{l}0.73 \pm \\
0.18\end{array}$ & & $\begin{array}{l}0.89 \pm \\
0.11\end{array}$ & & $\begin{array}{l}71.3 \pm \\
20.8\end{array}$ & \\
\hline \multicolumn{7}{|c|}{$\begin{array}{l}\text { SF-6D = Short-Form }-6 \text { dimensions. EQ-5D = European quality of life }-5 \text { dimensions. EQ-VAS }= \\
\text { Europen quality of life-visual analog scale. } \pm \text { sd }=\text { standard deviation. BMI = Body mass index. Bold } \\
\text { indicates P values }<0.05 \text { (one-way ANOVA, Kruskal-Wallis, independent sample t-test or Mann- } \\
\text { Whitney test). Different letters indicate significant differences between categories using Bonferroni's } \\
\text { or Dunnett's multiple comparison test (P values }<0.05 \text { ). }\end{array}$} \\
\hline
\end{tabular}

The relationship between autosomal ancestry and HRQoL was analyzed (Table 5). The proportion of Native America, European and African ancestries were categorized as terciles. The autosomal ancestry terciles showed no significant differences in HRQoL indexes. 
Table 5

Association between autosomal ancestry and health-related quality of life in type 1 diabetes patients.

\begin{tabular}{|c|c|c|c|c|c|c|}
\hline \multirow[t]{2}{*}{ Autosomal ancestry } & SF-6D & \multirow[t]{2}{*}{$P$ value } & EQ-5D & \multirow[t]{2}{*}{$P$ value } & EQ-VAS & \multirow[t]{2}{*}{$P$ value } \\
\hline & mean $\pm s d$ & & mean $\pm s d$ & & mean $\pm s d$ & \\
\hline \multicolumn{2}{|l|}{ Native American } & \multicolumn{2}{|l|}{0.256} & \multicolumn{2}{|l|}{0.554} & \multirow[t]{2}{*}{0.305} \\
\hline 1 st tertile & \multicolumn{2}{|l|}{$0.79 \pm 0.20$} & $0.91 \pm 0.09$ & & $79.4 \pm 20.9$ & \\
\hline 2nd tertile & \multicolumn{2}{|l|}{$0.73 \pm 0.21$} & $0.89 \pm 0.11$ & & \multicolumn{2}{|l|}{$73.4 \pm 19.1$} \\
\hline 3rd tertile & \multicolumn{2}{|l|}{$0.76 \pm 0.14$} & $0.90 \pm 0.10$ & & \multicolumn{2}{|l|}{$74.2 \pm 23.1$} \\
\hline \multicolumn{2}{|l|}{ European } & \multicolumn{2}{|l|}{0.919} & 0.524 & & \multirow[t]{2}{*}{0.294} \\
\hline 1 st tertile & \multicolumn{2}{|l|}{$0.75 \pm 0.17$} & $0.89 \pm 0.10$ & & $71.7 \pm 23.9$ & \\
\hline 2nd tertile & \multicolumn{2}{|l|}{$0.76 \pm 0.19$} & $0.90 \pm 0.11$ & & \multicolumn{2}{|l|}{$77.6 \pm 19.8$} \\
\hline 3rd tertile & \multicolumn{2}{|l|}{$0.76 \pm 0.19$} & $0.91 \pm 0.09$ & & \multicolumn{2}{|l|}{$77.4 \pm 19.7$} \\
\hline \multicolumn{2}{|l|}{ African } & \multicolumn{2}{|l|}{0.642} & 0.510 & & 0.805 \\
\hline 1 st tertile & $0.77 \pm 0.18$ & & $0.91 \pm 0.09$ & & \multicolumn{2}{|l|}{$77.1 \pm 19.2$} \\
\hline 2nd tertile & \multicolumn{2}{|l|}{$0.76 \pm 0.17$} & $0.90 \pm 0.10$ & & \multicolumn{2}{|l|}{$74.3 \pm 23.4$} \\
\hline 3rd tertile & \multicolumn{2}{|l|}{$0.74 \pm 0.20$} & $0.89 \pm 0.11$ & & \multicolumn{2}{|l|}{$75.5 \pm 21.0$} \\
\hline \multicolumn{7}{|c|}{$\begin{array}{l}S F-6 D=\text { Short-Form }-6 \text { dimensions. EQ }-5 D=\text { European quality of life }-5 \text { dimensions. EQ-VAS }= \\
\text { Europen quality of life-visual analog scale. } \pm \text { sd }=\text { standard deviation. } P \text { values calculated by one- } \\
\text { way ANOVA or Kruskal-Wallis test. }\end{array}$} \\
\hline
\end{tabular}

In addition, significant differences were found in proportion of European and African ancestries according to self-reported color/race $(P<0.001)$. Self-reported whites had higher European ancestry, and selfreported blacks had higher African ancestry. A statistically higher European ancestry was observed in patients with glycated hemoglobin $A 1 c \geq 7 \%(P=0.035)$. Diabetic retinopathy and kidney damage had no association with proportion of autosomal ancestry in the evaluated sample (Table 6). 
Table 6

Proportions of African, European and Native American ancestry according to self-reported race/color and clinical outcomes.

\begin{tabular}{|c|c|c|c|c|c|c|}
\hline \multirow[t]{2}{*}{ Autosomal ancestry } & $\begin{array}{l}\text { Native } \\
\text { American }\end{array}$ & \multirow[t]{2}{*}{$\begin{array}{l}P \\
\text { value }\end{array}$} & European & \multirow[t]{2}{*}{$\begin{array}{l}P \\
\text { value }\end{array}$} & African & \multirow[t]{2}{*}{$\begin{array}{l}P \\
\text { value }\end{array}$} \\
\hline & mean $\pm s d$ & & mean $\pm s d$ & & mean $\pm s d$ & \\
\hline Self-reported color/race & & 0.303 & & $<0.001$ & & $<0.001$ \\
\hline White & $0.24 \pm 0.09$ & & $\begin{array}{l}0.53 \pm \\
0.13^{\mathrm{a}}\end{array}$ & & $\begin{array}{l}0.22 \pm \\
0.10^{\mathrm{a}}\end{array}$ & \\
\hline Black & $0.20 \pm 0.11$ & & $\begin{array}{l}0.29 \pm \\
0.11^{\mathrm{b}}\end{array}$ & & $\begin{array}{l}0.50 \pm \\
0.14^{\mathrm{b}}\end{array}$ & \\
\hline Brown & $0.25 \pm 0.09$ & & $\begin{array}{l}0.45 \pm \\
0.13^{c}\end{array}$ & & $\begin{array}{l}0.29 \pm \\
0.11^{c}\end{array}$ & \\
\hline $\begin{array}{l}\text { Glycated hemoglobin } \\
\text { A1c }\end{array}$ & & 0.095 & & 0.035 & & 0.210 \\
\hline$<7 \%$ & $0.27 \pm 0.10$ & & $\begin{array}{l}0.41 \pm \\
0.14\end{array}$ & & $0.31 \pm 0.15$ & \\
\hline$\geq 7 \%$ & $0.24 \pm 0.09$ & & $\begin{array}{l}0.47 \pm \\
0.14\end{array}$ & & $0.27 \pm 0.12$ & \\
\hline Diabetic Retinopathy & & 0.075 & & 0.262 & & 0.937 \\
\hline Absent & $0.23 \pm 0.08$ & & $\begin{array}{l}0.48 \pm \\
0.15\end{array}$ & & $0.28 \pm 0.14$ & \\
\hline Present & $0.28 \pm 0.10$ & & $\begin{array}{l}0.42 \pm \\
0.15\end{array}$ & & $0.28 \pm 0.10$ & \\
\hline Kidney damage & & 0.659 & & 0.310 & & 0.410 \\
\hline Absent & $0.24 \pm 0.09$ & & $\begin{array}{l}0.47 \pm \\
0.14\end{array}$ & & $0.28 \pm 0.13$ & \\
\hline Present & $0.25 \pm 0.08$ & & $\begin{array}{l}0.44 \pm \\
0.15\end{array}$ & & $0.30 \pm 0.11$ & \\
\hline $\begin{array}{l} \pm s d=\text { standard deviatio } \\
\text { significant differences b } \\
0.05 \text { ). }\end{array}$ & $\begin{array}{l}\text { values cal } \\
\text { veen catego }\end{array}$ & $\begin{array}{l}\text { by ol } \\
\text { ing Bo }\end{array}$ & $\begin{array}{l}\text { ay ANOVA } \\
\text { roni's mul }\end{array}$ & $\begin{array}{l}\text { ifferent } \\
\text { compa }\end{array}$ & $\begin{array}{l}\text { ters indicate } \\
\text { on test ( } P \text { val }\end{array}$ & es $<$ \\
\hline
\end{tabular}

\section{Discussion}

This study is the first to assess the quality of life of patients with T1D in the State of Maranhão, an admixed population of the Northeast region of Brazil. In our evaluation, the variables that positively influenced the HRQoL of these patients included the following: being male, age under 18 years old, single 
status, elementary school education, having health insurance, having less than FIVE years of diagnosis, and practicing certain types of physical activity. In addition, non-occurrence of hypoglycemia in the last 30 days, lack of chronic complications (retinopathy and nephropathy), participation in any group educational activity, using analogous insulin, monitoring blood glucose, maximum adherence to treatment, and coming from secondary service showed statistical significance. Autosomal ancestry and self-reported color/race did not show influence on HRQoL indexes.

In a multicenter national study conducted in 2015 , the average score attributed to general health (EQ-VAS) was lower than that obtained in the present study $(72.5 \pm 22$ versus $76.24 \pm 21.30)$. The differences found in the literature in relation to the quality of life are related to urbanization, poverty, and organization of health services. However, despite this structure, the study mentioned above showed better quality of life reported in the EQ-VAS $(74.6 \pm 30)$, lower depression rate, and lower anxiety frequency in the northeast region, which characteristically features a lower urbanization rate, wealth, and structuring in health services. These findings were like those observed in our study, suggesting additional factors, such as lifestyle, in this assessment (17)

When we associated the socio-demographic criteria with the quality of life through HRQoL, the male patients showed better HRQoL. Other studies have already observed the lower quality of life among female patients (3)(18)(19). Women generally present higher disease-related concerns, lower level of satisfaction, and worse perception of their health compared with men (19).

We observed significant improvement in the HRQoL in the group with supplementary health insurance. The American Diabetes Association observed that patients with public and private health insurance have more access to health care and thus achieve better glycemic indexes and quality of life (9). A recent study evaluating a significant sample of the Brazilian population corroborates our findings of fewer diabetesrelated chronic complications, especially in retinopathy, when patients have access to public and private health services(20).

We also observed better HRQoL in the patients who participated in group education in secondary care. A study in the United Kingdom evaluated the HRQoL before and after a three-day educational course offered to adolescents with T1D to help manage diabetes. The results showed that the group's pre-course yearnings were met, and the educational assessment was solid. The A1c indices and BMI were unchanged, and no episodes of hypoglycemia were observed; both parents and patients reported an improved HRQoL after training (21). In a Greek study, patients with T1D participated for 1 year in groups, in which knowledge about the disease was transmitted in a simple and understandable manner. At the end of this program, reduced A1c levels, fewer blood glucose fluctuations, and lower incidence of hypoglycemia were observed, improving the HRQoL of these patients (22).

The use of analog insulin also demonstrated a positive impact on the HRQoL of our population. In the literature, this finding is controversial. A 2018 meta-analysis showed no overall difference in HRQoL compared with the use of the recombinant human insulin (NPH) (23). A Brazilian meta-analysis in 2019 failed to reach a consensus on the superiority of using fast acting analogs over regular insulin due to the 
scarcity of well-designed studies in the literature (24). However, reductions in severe hypoglycemia, postprandial blood glucose, and HBA1c, factors that impact the quality of life, were observed.

HBA1 $\mathrm{c}$ is an indirect measure of mean blood glucose levels, reflecting blood glucose levels over the past three months; despite its known limitations, it remains the primary tool for ensuring glycemic control and predicting the risk of complications (25). The mean HBA1c in this study was slightly lower than that observed in a multicenter Brazilian study in 2015, specifically in terms of the overall average of Brazil $(9.05 \pm 2.27$ versus $9.4 \pm 2.4)$ and in the evaluation of the northeast region of Brazil $(9.05 \pm 2.27$ versus $9.6 \pm 2.6$ ). No significant difference in HRQoL was observed between the patients with or without good metabolic control as evaluated by this tool. We attribute this finding to the large number of patients without good metabolic control, that is, $82.39 \%$ of our population. In a cohort from the diabetes outpatient clinic of the Hospital das Clinicas of the Federal University of Paraná with T1D adolescents, the patients with the best HRQoL included those with lower HBA1c levels; the higher the HBA1c, the greater the likelihood of lower levels of satisfaction (26). A previous study assessing adolescents with T1D observed that those with A1c in their goal may realize that diabetes results in an unfavorable effect on their lives, resulting in depression and difficulty in coping with the disease (27).

Physical activity is related to patients' lifestyle and is an impact variable in diabetes care. Every patient should be encouraged to allot leisure periods of physical activity and balanced exercise (9). In this study, sedentary lifestyle exhibited a significant negative impact on the HRQoL, in line with other studies conducted on patients with T1D and HRQoL (18)(26). Physical activity is strongly associated with psychological well-being and therefore, should be encouraged in this population (28).

Hypoglycemia, another important factor in the evaluation of this group, is described as the main limiting factor in the management of T1D (9). The data obtained in our research regarding the frequency hypoglycemia episodes and the fear of these episodes are in line with other studies demonstrating impairment in HRQoL (29). Hypoglycemia has been associated with cognitive dysfunction in children with T1D, and the fear of hypoglycemia may add to the difficulty faced by patients in adhering to the proposed treatment and therefore glycemic control (30)(31).

According to the literature, insufficient diabetes control and increased BMI negatively influence HRQoL, because they generate emotional disorders, such as anxiety, anguish, depression, low self-esteem, anorexia, or bulimia, whereas adequate capillary blood glucose monitoring and dietary flexibility are related to higher levels of HRQoL (19). We observed no difference in the HRQoL between the patients with different BMI levels. However, capillary blood glucose monitoring showed improvement in the HRQoL of patients.

A survey of young Germans comparing the HRQoL of T1D adolescents aged 11 to 17 years old and their healthy peers showed that the diagnosis of the disease in early childhood caused no impairment in the HRQoL compared with that of the peers without diabetes. This finding is probably due to the process of adaptation common in individuals with chronic diseases, with those who experienced such process from an early age becoming accustomed to their condition, considering their disease as normal and as a part 
of their daily life; as a result, and individuals with chronic diseases feel no different from their healthy peers (32). A Brazilian study showed by linear regression that complications and time of diabetes had low impact on EQ-VAS and failed to clarify the causes (16). Our study failed to show a better HRQoL of patients when considering the same variables. With earlier diagnosis of T1D, a better EQ-VAS was observed in patients with less disease time, and this finding is related to the absence of complications.

The presence of microvascular complications (retinopathy and nephropathy) was associated with a lower HRQoL in our study. The 23-year study of DCCT/DTIS also showed that the presence of microvascular complications significantly decreased the HRQoL in patients with T1D (33). Retinopathy and nephropathy impair the autonomy, self-care, and HRQoL of patients with T1D (34).

Our study has shown a better HRQoL in patients coming from the secondary public service, which can be attributed to the higher number of patients using insulin analogs and presenting less hypoglycemia, which are important factors affecting the HRQoL of our patients. In addition, these patients had health insurance and received various services in private services, which may also influence their perception of HRQoL. These patients also had more access to educational programs, which have been shown by several studies to positively impact HRQoL (21)(22).

We found a negative correlation between European ancestry and glycemic control, the higher the degree of European ancestry the worse HBA1C. Evidence suggests that minority populations tend to have poorer self- management and diabetes outcomes, for example African and Hispanic children and adolescents have worse control when compared to their white peers (10)(35). We hypothesize that the difference in our study is due to the large percentage of European ancestry found in our highly mixed population.

Our population is composed by the miscegenation between European, African, and Native American populations (36). This fact was noted in our study through the analysis of genomic ancestry. Through our analyses we found that, as in all Brazilian regions, European ancestry was the largest contributor, but in our population, it approached $50 \%$ differing from the weighted average of $68.1 \%$ found in the Brazilian population in a systematic review study conducted in 2019. We also obtained a similar percentage between African and Native American ancestry (around 25\% each), which again differs from the Brazilian average of $19.6 \%$ African and $11.6 \%$ Native American. The distribution of ancestral groups did not occur homogeneously in the Brazilian territory, differing depending on the geographic region, and reflecting the history of colonization with different levels of miscegenation (37). In general, this occurred with an asymmetrical mating pattern, preferably between European men and Native American or African women. In Afro-descendant communities such as the Amazon and Maranhão, another pattern between African men and Native American women was also observed (36). Also, in Maranhão, Native Americans maintained contact with the Brazilian population of mixed race and with African slaves, in the pattern of the mating of Native American men with African or mixed women (38). These patterns may justify our observed ancestry panel.

Surveys have shown a low correlation between color report and ancestry, however, in our analysis we found that in self-reported whites had higher European ancestry, and self-reported blacks had higher 
African ancestry, suggesting adequate perception by our patients (39).

Quality of life questionnaires are barely explored in Brazil, particularly in the State of Maranhão, the northeast region of the country. Our study is the first to measure HRQoL and recognize the impact of T1D through the analysis of multiple factors related to the quality of life and autosomal ancestry in patients with from Maranhão, a state formed by a population highly admixed. The results validate the need to provide T1D patients with continuous training on self-management and self-monitoring, seeking better results in metabolic control and consequently, in the prevention of acute and chronic complications to generate positive impacts on the quality of life of this population. In addition, reinforcing physical activity at each appointment should be part of the health team's routine.

\section{Declarations}

\section{Availability of data and materials}

The datasets generated during the current study are available from the corresponding author on request.

\section{Author contributions}

Conceptualization: DL, GS, RA, MT, RR, NN.

Data curation: DL, GS, RA, MT, RR, NN, AA, AS.

Formal analysis: DL, GS, RA, VR, MT, RR, NN, AA, AS, MG, MF.

Funding acquisition: RA, MG, MF.

Methodology: DL, GS, RA, VR, MT, RR, NN, AA, AS, DS, MF.

Project administration: DL, RA, MT, RR, NN, AA, AS, MF.

Visualization: DL, MM, GN, SD, VR, MF, RA.

Writing - original draft: DL, GS, RA.

Writing - review \& editing: DL, GS, RA, VR, MT, MM, RR, NN, AA, AS, GN, SD, VR, MG, MF.

\section{ORCID}

1. Rossana Azulay, ORCID: 0000-0002-6097-8145

2. Débora Lago, ORCID: 0000-0002-9311-5047

3. Glaucia Abreu Silva Santos, ORCID: 0000-0002-4451-472X 
4. Maria da Gloria Tavares, ORCID: 0000-0001-6531-0227

5. Vandilson Rodrigues, ORCID: 0000-0002-6785-7864

6. Marcelo Magalhães, ORCID: 0000-0002-0464-4343

7. Roberta Ferreira Reis, ORCID: 0000-0002-0940-4357

8. Nayara Nunes, ORCID: 0000-0002-6578-7436

9. Ana Gregória Almeida, ORCID: 0000-0002-4049-4629

10. Adriana Guimarães Sá, ORCID: 0000-0003-1963-8816

11. Gilvan Nascimento, ORCID: 0000-0002-3373-4372

12. Sabrina Damianse, ORCID: 0000-0001-6531-8122

13. Viviane Rocha, ORCID: 0000-0002-2498-3289

14. Dayse Aparecida Silva, ORCID: 0000-0003-3805-2837

15. Marília Brito Gomes, ORCID: 0000-0003-4458-4741

16. Manuel Faria, ORCID: 0000-0003-0732-668X

\section{Funding}

This work was supported in part by the Research Support Foundation of Maranhão State (FAPEMA) and the Coordenação de Aperfeiçoamento de Pessoal de Nível Superior Brasil (CAPES).

\section{Ethics declarations}

\section{Ethics approval and consent to participate}

The study protocol was approved by the Research Ethics Committee of the University Hospital of the Federal University of Maranhão-CEP/HU-UFMA (protocol 23523.004536/2016-76).

\section{Consent to participate}

Informed consent was obtained from all individual participants included in the study.

\section{Consent for publication}

All authors have consented to submission of this article for publication.

\section{Competing interests}

The authors declare that they have no competing interests.

\section{References}

1. Sitlinger A, Yousuf Zafar S. Health-Related Quality of Life: The Impact on Morbidity and Mortality. Surg Oncol Clin N Am. 2018;27:675-84. 
2. Lima L. Qualidade de vida de pacientes com diabetes mellitus tipo 1. Vol. 1. 2009.

3. Cruz DSM da, Collet N, Nóbrega VM. Qualidade de vida relacionada à saúde de adolescentes com dm1- revisão integrativa. Cien Saude Colet [Internet]. 2018 Mar;23(3):973-89. Available from: http://www.scielo.br/scielo.php?script=sci_arttext\&pid=S1413$81232018000300973 \&$ lng=pt\&tlng=pt.

4. Negrato CA, Dias JPL, Teixeira MF, Dias A, Salgado MH, Lauris JR, et al. Temporal trends in incidence of Type 1 diabetes between 1986 and 2006 in Brazil. J Endocrinol Invest [Internet]. 2010 Jun 20;33(6):373-7. Available from: http://link.springer.com/10.1007/BF03346606.

5. Lyra R, Azevedo Junior LGG, Diniz ET, Ibiapina GR, Veloso IGLFK, et al. Diabetes melito: classificação e diagnóstico. Endocrinologia clínica. 6. ed. Rio de Janeiro: Guanabara Koogan; 2016. pp. 641-56.

6. Wagner VM, Müller-Godeffroy E, von Sengbusch S, Häger S, Thyen U. Age, metabolic control and type of insulin regime influences health-related quality of life in children and adolescents with type 1 diabetes mellitus. Eur J Pediatr [Internet]. 2005 Aug 5;164(8):491-6. Available from: http://link.springer.com/10.1007/s00431-005-1681-4.

7. de Wit M, Delemarre-van de Waal HA, Bokma JA, Haasnoot K, Houdijk MC, Gemke RJ, et al. Selfreport and parent-report of physical and psychosocial well-being in Dutch adolescents with type 1 diabetes in relation to glycemic control. Health Qual Life Outcomes [Internet]. 2007 Dec 16;5(1):10. Available from: https://hqlo.biomedcentral.com/articles/10.1186/1477-7525-5-10.

8. Sá JR, Canani LH, Rangel EB, Bauer AC, Escott, Gustavo Monteiro Zelmanovitz T, Silveiro S, Bertoluci M. Doença renal do diabetes [Internet]. Diretriz SBD 2021. 2021. Available from: https://diretriz.diabetes.org.br/doenca-renal-do-diabetes/.

9. Introduction: Standards of Medical Care in Diabetes-2019. Diabetes Care [Internet]. 2019 Jan 17;42(Supplement 1):S1-2. Available from: http://care.diabetesjournals.org/lookup/doi/10.2337/dc19-Sint01.

10. Kane NS, Hoogendoorn CJ, Commissariat PV, Schulder TE, Gonzalez JS. Glycemic control and selfrated health among ethnically diverse adolescents with type 1 diabetes. Pediatr Diabetes [Internet]. 2020 Feb 12;21(1):69-76. Available from: https://onlinelibrary.wiley.com/doi/10.1111/pedi.12928.

11. EuroQol - a new facility for the measurement of health-related quality of life. Health Policy (New York) [Internet]. 1990 Dec;16(3):199-208. Available from: https://linkinghub.elsevier.com/retrieve/pii/0168851090904219.

12. Núcleo de Avaliação de Tecnologias em Saúde (NATS-INC) [Internet]. 2021. Available from: http://natsinc.org/wpress/euroqol/.

13. Cruz LN, Camey SA, Hoffmann JF, Rowen D, Brazier JE, Fleck MP, et al. Estimating the SF-6D Value Set for a Population-Based Sample of Brazilians. Value Heal [Internet]. 2011 Jul;14(5):S108-14. Available from: https://linkinghub.elsevier.com/retrieve/pii/S1098301511014288.

14. Campolina AG, Bortoluzzo AB, Ferraz MB, Ciconelli RM. Validação da versão brasileira do questionário genérico de qualidade de vida short-form 6 dimensions (SF-6D Brasil). Cien Saude Colet 
[Internet]. 2011 Jul;16(7):3103-10. Available from: http://www.scielo.br/scielo.php? script=sci_arttext\&pid=S1413-81232011000800010\&lng=pt\&tlng=pt.

15. KDIGO 2020 Clinical Practice Guideline for Diabetes Management in Chronic Kidney Disease. Kidney Int [Internet]. 2020;98(45). Available from: https://kdigo.org/wp-content/uploads/2020/10/KDIGO2020-Diabetes-in-CKD-GL.pdf.

16. Pereira R, Phillips C, Pinto N, Santos C, dos Santos SEB, Amorim A, et al. Straightforward inference of ancestry and admixture proportions through ancestry-informative insertion deletion multiplexing. PLoS One. 2012;7(1).

17. Felício JS, De Souza ACCB, Koury CC, Neto JFA, Miléo KB, Santos FM, et al. Health-related quality of life in patients with type 1 diabetes mellitus in the different geographical regions of Brazil: Data from the Brazilian Type 1 Diabetes Study Group. Diabetol Metab Syndr. 2015;7(1).

18. Santos DC, Pizarro MH, Barros BSV, de Melo LGN, Porto LC, Silva DA, et al. Does ancestry influence health-related quality of life in type 1 diabetes patients? A nationwide study in Brazil. Acta Diabetol [Internet]. 2018;55(4):377-85. Available from: https://doi.org/10.1007/s00592-017-1096-5.

19. Matziou V, Tsoumakas K, Vlahioti E, Chrysicopoulou L, Galanis P, Petsios K, et al. Factors influencing the quality of life of young patients with diabetes. J Diabetes [Internet]. 2011 Mar;3(1):82-90. Available from: https://onlinelibrary.wiley.com/doi/10.1111/j.1753-0407.2010.00106.x.

20. Gomes MB, Santos DC, Pizarro MH, Melo LGN, Barros BSV, Montenegro R, et al. Relationship between health care insurance status, social determinants and prevalence of diabetes-related microvascular complications in patients with type 1 diabetes: a nationwide survey in Brazil. Acta Diabetol [Internet]. 2019 Jun 13;56(6):697-705. Available from: http://link.springer.com/10.1007/s00592-019-01308-7.

21. Waller H, Eiser C, Knowles J, Rogers N, Wharmby S, Heller S, et al. Pilot study of a novel educational programme for 11-16 year olds with type 1 diabetes mellitus: the KICk-OFF course. Arch Dis Child [Internet]. 2008 Nov 1;93(11):927-31. Available from: https://adc.bmj.com/lookup/doi/10.1136/adc.2007.132126.

22. Mouslech Z, Somali M, Sarantis L, Christos D, Alexandra C, Maria P, et al. Significant effect of group education in patients with diabetes type 1. Hormones [Internet]. 2018 Sep 15;17(3):397-403. Available from: http://link.springer.com/10.1007/s42000-018-0054-0.

23. Almeida PHRF, Silva TBC, de Assis Acurcio F, Guerra Júnior AA, Araújo VE, Diniz LM, et al. Quality of Life of Patients with Type 1 Diabetes Mellitus Using Insulin Analog Glargine Compared with NPH Insulin: A Systematic Review and Policy Implications. Patient - Patient-Centered Outcomes Res [Internet]. 2018 Aug 10;11(4):377-89. Available from: http://link.springer.com/10.1007/s40271-0170291-3.

24. Melo KFS, Bahia LR, Pasinato B, Porfirio GJM, Martimbianco AL, Riera R, et al. Short-acting insulin analogues versus regular human insulin on postprandial glucose and hypoglycemia in type 1 diabetes mellitus: A systematic review and meta-analysis 11 Medical and Health Sciences 1103 Clinical Sciences. Diabetol Metab Syndr [Internet]. 2019;11(1):1-13. Available from: https://doi.org/10.1186/s13098-018-0397-3. 
25. Glycemic Targets: Standards of Medical Care in Diabetes-2020. Diabetes Care [Internet]. 2020 Jan 20;43(Supplement 1):S66-76. Available from:

http://care.diabetesjournals.org/lookup/doi/10.2337/dc20-S006.

26. Martins KAKF, Mascarenhas LPG, Morandini M, Cat MNL, Pereira RM, De Carvalho JR, et al. Healthrelated quality of life in a cohort of youths with type 1 diabetes. Rev Assoc Med Bras. 2018;64(11):1038-44.

27. Grey M, Boland EA, Yu C, Sullivan-Bolyai S, Tamborlane WV. Personal and Family Factors Associated With Quality of Life in Adolescents With Diabetes. Diabetes Care [Internet]. 1998 Jun 1;21(6):909-14. Available from: http://care.diabetesjournals.org/cgi/doi/10.2337/diacare.21.6.909.

28. Åman J, Skinner T, de Beaufort C, Swift P, Aanstoot H-J, Cameron F. Associations between physical activity, sedentary behavior, and glycemic control in a large cohort of adolescents with type 1 diabetes: the Hvidoere Study Group on Childhood Diabetes. Pediatr Diabetes [Internet]. 2009 Jun;10(4):234-9. Available from: https://onlinelibrary.wiley.com/doi/10.1111/j.13995448.2008.00495.x.

29. Schunk M, Reitmeir P, Schipf S, Völzke H, Meisinger C, Ladwig K-H, et al. Health-related quality of life in women and men with type 2 diabetes: a comparison across treatment groups. J Diabetes Complications [Internet]. 2015 Mar;29(2):203-11. Available from: https://linkinghub.elsevier.com/retrieve/pii/S1056872714003377.

30. Martyn-Nemeth P, Schwarz Farabi S, Mihailescu D, Nemeth J, Quinn L. Fear of hypoglycemia in adults with type 1 diabetes: impact of therapeutic advances and strategies for prevention - a review. J Diabetes Complications [Internet]. 2016 Jan;30(1):167-77. Available from: https://linkinghub.elsevier.com/retrieve/pii/S1056872715003736.

31. Rodrigues Vilela V, de Castro Ruiz Marques A, Schamber CR, Bazotte RB. Hypoglycemia Induced by Insulin as a Triggering Factor of Cognitive Deficit in Diabetic Children. Sci World J [Internet]. 2014;2014:1-9. Available from: http://www.hindawi.com/journals/tswj/2014/616534/.

32. Stahl A, Strassburger K, Lange K, Bachle C, Holl RW, Giani G, et al. Health-Related Quality of Life Among German Youths With Early-Onset and Long-Duration Type 1 Diabetes. Diabetes Care [Internet]. 2012 Aug 1;35(8):1736-42. Available from: http://care.diabetesjournals.org/cgi/doi/10.2337/dc11-2438.

33. Jacobson AM, Braffett BH, Cleary PA, Gubitosi-Klug RA, Larkin ME. The Long-Term Effects of Type 1 Diabetes Treatment and Complications on Health-Related Quality of Life: A 23-year follow-up of the Diabetes Control and Complications/Epidemiology of Diabetes Interventions and Complications cohort. Diabetes Care [Internet]. 2013 Oct 1;36(10):3131-8. Available from: http://care.diabetesjournals.org/cgi/doi/10.2337/dc12-2109.

34. Yu Y, Feng L, Shao Y, Tu P, Wu HP, Ding X, et al. Quality of life and emotional change for middle-aged and elderly patients with diabetic retinopathy. Int J Ophthalmol. 2013;6(1):71-4.

35. Campbell JA, Walker RJ, Smalls BL, Egede LE. Glucose control in diabetes: The impact of racial differences on monitoring and outcomes. Endocrine. 2012;42(3):471-82. 
36. Saloum de Neves Manta F, Pereira R, Vianna R, Rodolfo Beuttenmüller de Araújo A, Leite Góes Gitaí D, Aparecida da Silva D, et al. Revisiting the Genetic Ancestry of Brazilians Using Autosomal AIM-Indels. PLoS One. 2013;8(9):1-11.

37. de Souza AM, Resende SS, de Sousa TN, de Brito CFA. A systematic scoping review of the genetic ancestry of the brazilian population. Genet Mol Biol. 2019;42(3):495-508.

38. Leite D, Marinho ANR, Rodrigues-Carvalho C, Ribeiro-dos-Santos Â, Souza S, Leitão A, et al. Paleogenetic Studies in Guajajara Skeletal Remains, Maranhão State, Brazil. J Anthropol. 2014;2014:1-8.

39. Gomes MB, Calliari LE, Santos DC, Muniz LH, Porto LC, Silva DA, et al. Genomic ancestry and glycemic control in adolescents with type 1 diabetes: A multicenter study in Brazil. Pediatr Diabetes. 2020;21(5):727-34.

\section{Figures}


a

\section{Autosomal ancestry}
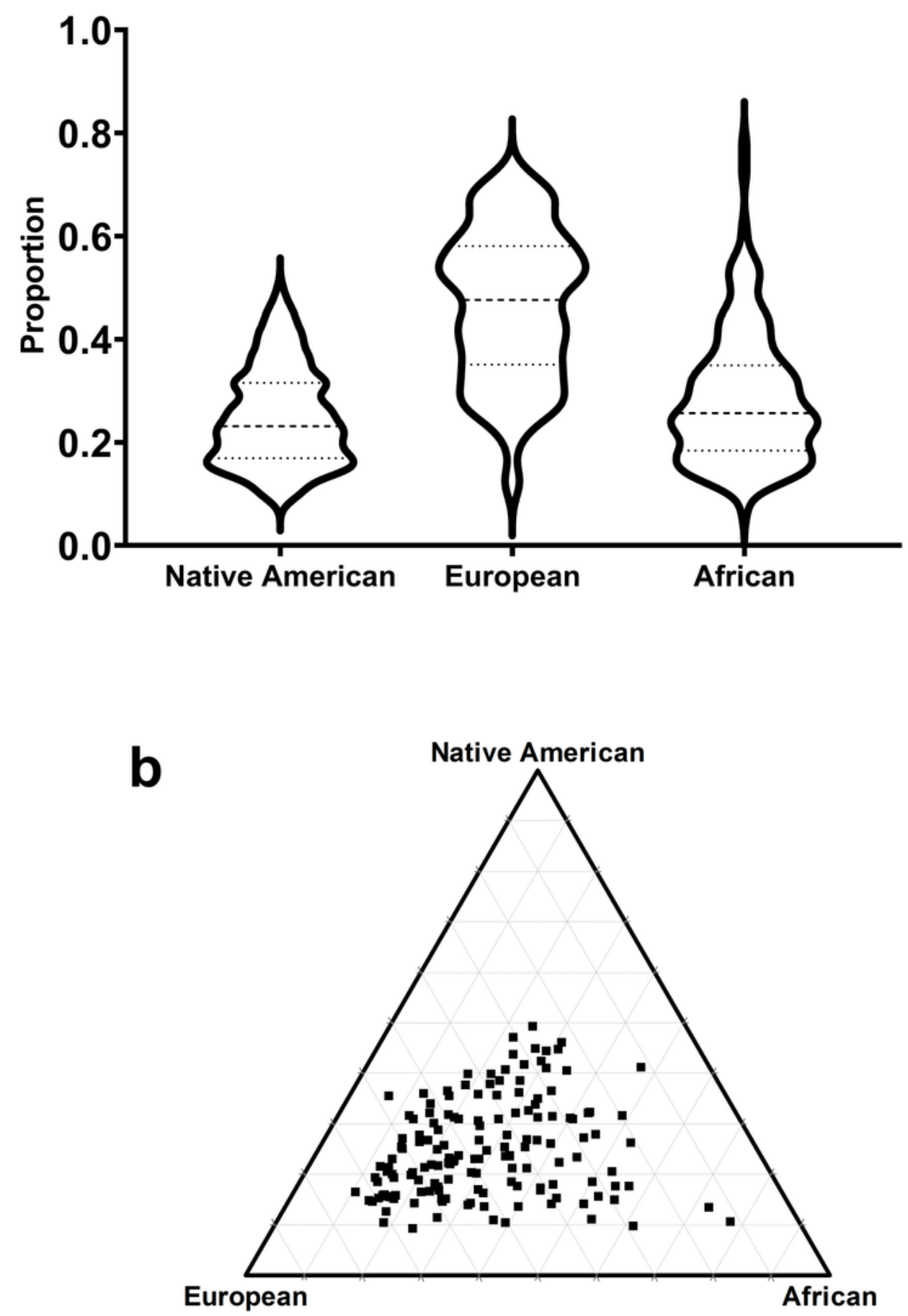

Figure 1

Violin plot (a) and triangular plot (b) of autosomal ancestry in type 1 diabetes patients. 